\title{
SEPARABLE ABELIAN GROUPS AS MODULES OVER THEIR ENDOMORPHISM RINGS
}

\author{
K. M. RANGASWAMY
}

\begin{abstract}
Properly separable mixed abelian groups $A$ which are projective, respectively flat, as modules over their endomorphism rings are completely characterized. These results generalize the works of F. Richman and E. A. Walker.
\end{abstract}

A subclass of separable mixed abelian groups - the properly separable groups - in which finitely many rank one summands can be embedded in a finite rank summand is considered here. It is shown that a reduced properly separable mixed abelian group $A$ is projective as a module over its endomorphism ring $E(A)$ exactly when $A=T \oplus\left(\bigoplus B_{i}\right), i \in I$, where $T$ is torsion with each of its $p$-components bounded, for each $i \in I, B_{i}$ contains a torsionfree summand of rank one whose type is idempotent and is the smallest in the typeset of $B_{i} /\left(B_{i}\right)_{t}$ and, finally, the subgroups $T$ and $B_{i}$ are all fully invariant in $A$. A result of F. Richman and E. A. Walker is generalised to show that the reduced properly separable mixed abelian groups $A$ which are $E(A)$-flat have the following characterising property: In the typeset of $A / A_{t}$, any nonempty finite subset has a lower bound whenever it has an upper bound.

All the groups that we consider here are additively written abelian groups. For the general notation and terminology, we refer to $[\mathbf{3}]$. By a group of rank one we mean a subgroup of rational numbers or a subgroup of the Prüfer group $Z\left(p^{\infty}\right)$ for some prime $p$. A mixed group $A$ is said to be separable if each nonempty finite subset can be embedded in a direct summand of $A$ which is a direct sum of finitely many rank one groups. For the general properties of separable mixed groups, we refer to [1 and 4]. As noted in [4], the height matrices of elements in a reduced separable mixed group have finitely many jumps and have as entries the nonnegative integers and the symbol $\infty$. We shall call such heights matrices separable. For two separable height matrices $H$ and $K$, define $H \sim K$, if (i) $H=K$, in case almost all the entries of $H$ are $\infty$, and (ii) $m H=n K$ for some positive integers $m, n$, in case $H$ has infinitely many finite entries. Then $\sim$ is an equivalence relation and each equivalence class is called a type. For two types $t_{1}$ and $t_{2}$, we define $t_{1} \geq t_{2}$ if there are height matrices $H_{1} \in t_{1}$ and $H_{2} \in t_{2}$ with $H_{1} \geq H_{2}$. Thus $t_{1} \geq t_{2}$ if $t_{1}$ is the height matrix type of an element of order $p^{n}$ and $t_{2}$ is that of a torsionfree element with finite $p$-height in $A$. For any reduced separable group $A$, let $\Gamma(A)$ denote the set of types of all its rank one summands.

A separable group $A$ is said to be properly separable if finitely many rank one

Received by the editors September 28, 1983. Presented at the 86th summer meeting of the American Mathematical Society at Toronto, August 25, 1982.

1980 Mathematics Subject Classification. Primary 20K21, $20 \mathrm{~K} 40$.

Key words and phrases. Abelian group, separable groups, projective and flat modules.

(c) 1984 American Mathematical Society $0002-9939 / 84 \$ 1.00+\$ .25$ per page 
summands can be embedded in a finite rank summand. Clearly, a separable group which is torsion or torsionfree is properly separable. As indicated in [4], if $A$ is properly separable and nontorison, then for each prime $p$ relevant to $A$, there is a rank one torsionfree summand whose type has 0 or $\infty$ as the $p$ th entry. We need the following result proved in [4]:

LEMMA 1. A properly separable reduced group $A$ decomposes as $A=T \oplus B$, where $T$ is torsion, $B$ is nontorsion with the property that, for each prime $p$ relevant to $B$, there is a $t \in \Gamma\left(B / B_{t}\right)$ whose pth entry is 0 and both $T$ and $B$ are fully invariant in $A$.

For later use, we say that a group $G$ has the Property (*) if it has the property of the group $B$ stated in Lemma 1 .

Our next theorem characterizes the properly separable groups $A$ which are flat over their endomorphism rings $E(A)$. F. Richman and E. A. Walker [6] described the torsionfree completely decomposable groups $A$ which are $E(A)$-flat. Our proof originated from theirs and the author is grateful to them for having given access to their preprint $[6]$.

THEOREM 2. Let $A$ be a reduced properly separable abelian group and let $E=$ $E(A)$. Then the following are equivalent:

(i) $A$ is E-flat.

(ii) For any two rank one summands $M_{1}, M_{2}$, whenever $\operatorname{Hom}\left(M_{1}, M_{3}\right) \neq 0$ and $\operatorname{Hom}\left(M_{2}, M_{3}\right) \neq 0$ for any rank one summand $M_{3}$, then there is a rank one summand $M_{4}$ such that $\operatorname{Hom}\left(M_{4}, M_{1}\right) \neq 0$ and $\operatorname{Hom}\left(M_{4}, M_{2}\right) \neq 0$.

(iii) In $\Gamma(A)$ any nonempty finite subset has a lower bound whenever it has an upper bound.

ProOF. (i) $\Rightarrow$ (ii). Let $0 \neq f_{1}: M_{1} \rightarrow M_{3}$ and $0 \neq f_{2}: M_{2} \rightarrow M_{3}$. Then $f_{1}\left(x_{1}\right)=$ $f_{2}\left(x_{2}\right) \neq 0$ for some $x_{i} \in M_{i}, i=1,2$. By the flatness of $A$, there exist $y_{j} \in$ $A, f_{i j} \in E$, for $i=1,2$ and $j=1, \ldots, t$, such that $x_{i}=\sum_{j=1}^{t} f_{i j} y_{j}$, for $i=1,2$ and $f_{1} f_{1 j}=f_{2} f_{2 j}$, for all $j=1, \ldots, t$ (see [2]). Then $f_{1} f_{1 j}\left(y_{j}\right)=f_{2} f_{2 j}\left(y_{j}\right) \neq 0$ for some $j$. Since $A$ is separable, $f_{1} f_{1 j}(z)=f_{2} f_{2 j}(z) \neq 0$ for some $z$ in a rank one summand $M_{4}$ of $A$. Then $\operatorname{Hom}\left(M_{4}, M_{1}\right) \neq 0 \neq \operatorname{Hom}\left(M_{4}, M_{2}\right)$.

(ii) $\Rightarrow$ (iii). Obvious.

(iii) $\Rightarrow$ (i). We first show that $A[\tau]$ is a flat $E$-module if $\tau$ is the type of a torsionfree summand $S$ of rank one in $A$. Let $\tau=\left(k_{1}, \ldots, k_{n}, \ldots\right)$. Note that $k_{i}=0$ or $\infty$ if the associated prime $p_{i}$ is relevant to $A$ (because $A$ is properly separable). Let $x_{0} \in S$ such that $x_{0}$ has characteristic $\left(k_{1}, \ldots, k_{n}, \ldots\right)$. Let $K=\left\{k_{i}: k_{i} \neq 0\right.$ or $\left.\infty\right\}$, $\left\{r_{1}, r_{2}, \ldots\right\}$ an enumeration of $K$, and $q_{1}, q_{2}, \ldots$ the corresponding primes. Define, for each $i, x_{i} \in S$ such that $q_{1}^{r_{1}} q_{2}^{r_{2}} \cdots q_{i}^{r_{i}} x_{i}=x_{0}$. Now, for any prime $p$ relevant to $A$, each $x_{i}$ has $p$-height 0 or $\infty$, so that if, for any $f \in E, f\left(x_{i}\right)=0$, then $f(S)=0$. If $e: A \rightarrow S$ is a projection, it then follows that for each $i, E e \simeq E x_{i}$ as an $E$-module under the map $f e \mapsto f\left(x_{i}\right)$. Since $A[\tau]$ is the union of the ascending chain of projective $E$-modules $E x_{0} \subset E x_{1} \subset E x_{2} \subset \cdots, A[\tau]$ is a flat $E$-module.

Now by Lemma $1, A=T \oplus B$, with $T$ torsion separable, $\oplus$ an $E$-module direct sum and $B$ having property $(*)$. By $[\mathbf{5}], T$ is $E(T)$-flat so we shall prove the result assuming that $A=B$. Let $\Gamma=\Gamma\left(A / A_{t}\right)$. For any $\tau_{1}, \tau_{2} \in \Gamma$, define $\tau_{1} \sim \tau_{2}$ if there is a $\tau \in \Gamma$ such that $\tau \leq \tau_{1}, \tau_{2}$. By hypothesis, $\sim$ is an equivalence relation. Let 
$\left\{\xi_{m}: m \in M\right\}$ be a partitioning of $\Gamma$ induced by $\sim$. Let, for each $m \in M, A_{m}=$ $\bigcup\left\{A[\tau]: \tau \in \xi_{m}\right\}$. Clearly $\operatorname{Hom}\left(A_{m}, A_{n}\right)=0$, for any $m, n \in M$ with $m \neq n$. The properties of $A$ then imply that $A$ is a direct sum of the $A_{m}$ 's. The result follows if one notes that each $A_{m}$, being a directed union of the flat $E$-modules $\{A[\tau]\}, \tau \in \xi_{m}$, is itself $E$-flat.

REMARK. From the first part of the proof of (iii) $\Rightarrow(\mathrm{i})$, we note the following: If $A$ is an arbitrary torsionfree abelian group and $\tau$ is the type of a rank one summand of $A$, then $A[\tau]$ is $E(A)$-flat.

We shall now consider the properly separable groups which are projective over their endomorphism rings. For any type $\tau$, let $\tau_{0}$ denote the reduced type of $\tau$ obtained by replacing the finite entries of $\tau$ by 0 . The reduced inner type $\sigma$ of a separable group $A$ is defined by $\sigma=\min \left\{\tau_{0}: \tau \in \Gamma(A)\right\}$.

We begin with the following useful lemma.

LEMMA 3. Suppose $A$ is a reduced nontorsion properly separable group. Suppose any two $\tau_{1}, \tau_{2}$ in $\Gamma(A)$ have a lower bound in $\Gamma(A)$. Then the center of $E(A)$ is a subring $R_{0}$ of rational numbers whose type is the reduced inner type of $A$.

PROOF. Clearly the multiplication by any rational number $r \in R_{0}$ acts as an endomorphism of $A$ and is in the center of $E(A)$. Conversely, let $f$ be an element of the center of $E(A)$. Then $f$ acts as an endomorphism of each rank one summand of $A$ and hence as a multiplication by a rational number $r$ whose type has entries 0 at those primes $p$ at which the type of some rank one summand of $A$ has a finite entry. Hence $r \in R_{0}$.

We are now ready to characterize those separable mixed groups $A$ which are $E(A)$-projective. These turn out to be direct sums of (cyclic) summands of $E(A)$.

THEOREM 4. Let $A$ be a reduced properly separable mixed abelian group and let $E=E(A)$. Then $A$ is projective as an $E$-module if and only if $A=T \oplus\left(\bigoplus B_{i}\right), i \in$ $I$, where $T$ and the $B_{i}$ are fully invariant, $T$ is torsion with each of its $p$-components bounded and, for each $i \in I, B_{i}$ contains a rank one torsionfree summand whose type is the reduced inner type of $B_{i}$.

Proof. Suppose $A$ is $E$-projective. By Lemma 1 , we have an $E$-module decomposition $A=T \oplus B$, with $T$ torsion and $B$ having property (*). Since $T$ is torsion and $E(T)$-projective, $T_{p}$ is bounded for each $p[5]$. Since $B$ is, in particular, $E(B)$-flat, it follows from the proof of Theorem 2 , that $B=\bigoplus B_{i}, i \in I$, where $\bigoplus$ is an $E$-module direct sum and, for each $i \in I, \Gamma\left(B_{i}\right)$ is directed below. For convenience in writing, let us replace a nontorsion $B_{i}$ by $A$. By the assumption on $B_{i}, A$ is a directed union of $\{A[\tau]: \tau \in \Gamma(A)\}$. Since $A$ is $E$-projective, there exists, by the dual basis theorem $[2], f_{j} \in \operatorname{Hom}_{E}(A, E), x_{j} \in A$ with $j \in J$, such that, for any $x \in A, f_{j}(x)=0$ for almost all $j \in J$ and $x=\sum f_{j}(x) x_{j}$. Then the $Z$-morphism

$$
\alpha: \operatorname{Hom}_{E}(A, E) \otimes_{E} A \rightarrow \operatorname{Hom}_{E}(A, A)
$$

given by $\alpha(f \otimes a)(x)=f(x) a$ is nonzero since $\alpha\left(\sum\left(f_{j} \otimes x_{j}\right)\right)(x)=\sum f_{j}(x) x_{j}=x$. As $A$ is a directed union of $A[\tau], \tau \in \Gamma(A), \alpha\left(\operatorname{Hom}_{E}(A, E) \otimes A[\tau]\right) \neq 0$ for some $\tau \in \Gamma(A)$. By Lemma $3, \operatorname{Hom}_{E}(A, A)=R_{0}$, the subring of rational numbers containing 1 and having the reduced inner type $\sigma_{0}$ of $A$. It is then readily seen that 
$\tau \leq \sigma_{0}$. Thus $A$ has a rank one torsionfree summand whose type is the reduced inner type of $A$.

Conversely, let $A=T \oplus\left(\bigoplus B_{i}\right), i \in I$, with $T$ torsion having all its $p$-components bounded and the $B_{i}$ having the stated properties. Let $\gamma_{i}: A \rightarrow B_{i}, i \in I$, be the projections corresponding to the given decomposition. If each $B_{i}$ contains a rank one summand $S_{i}$ with $e_{i}=e_{i} \gamma_{i}: A \rightarrow S_{i}$ a corresponding projection and $S_{i}$ having an element $x_{i}$ whose height matrix $=$ the reduced inner type of $B_{i}$, then the map $f e_{i} \mapsto f\left(x_{i}\right)$ is an $E$-isomorphism of $E e_{i}$ with $B_{i}$. Thus each $B_{i}$ is $E$-projective. Since, by [5], $T$ is $E$-projective, so is $A$.

\section{BIBLIOGRAPHY}

1. H. Bowman and K. M. Rangaswamy, On special balanced subgroups of torsionfree separable abelian groups, Abelian Group Theory (Proc. Oberwolfach Conf., 1981), Lecture Notes in Math., vol. 874, Springer-Verlag, Berlin and New York, 1981, pp. 32-40.

2. E. Cartan and S. Eilenberg, Homological algebra, Princeton Univ. Press, Princeton, N.J., 1956.

3. L. Fuchs, Infinite abelian groups. II, Academic Press, New York, 1973.

4. K. M. Rangaswamy, The theory of separable mixed abelian groups (to appear).

5. F. Richman and E. A. Walker, Primary abelian groups as modules over their endomorphism rings, Math. Z. 89 (1965), 77-81.

6. __ Cyclic Ext (preprint).

Department of Mathematics, College of Engineering \& Applied Science, UNIVERSITY OF COLORADO, COLORADO SPRINGS, COLORADO 80933-7150 